\title{
Shape and Size Effects in the Crystal Structures of Complexes of 1,3,5-Trinitrobenzene with some Trigonal Donors: The Benzene-Thiophene Exchange Rule
}

\author{
Praveen K. Thallapally, ${ }^{a}$ Kakali Chakraborty, ${ }^{\mathrm{b}}$ H. L. Carrell, ${ }^{\mathrm{c}}$ Sambasivarao Kotha ${ }^{\mathrm{b}}$ and \\ Gautam R. Desiraju ${ }^{\mathrm{a}, *}$ \\ ${ }^{a}$ School of Chemistry, University of Hyderabad, Hyderabad 500 046, India \\ ${ }^{\mathrm{b}}$ Department of Chemistry, Indian Institute of Technology, Powai, Mumbai 400 076, India \\ ${ }^{\mathrm{c}}$ The Institute for Cancer Research, Fox Chase Cancer Center, 7701 Burholme Avenue, Philaldelphia, PA 19111, USA
}

\begin{abstract}
The crystal chemistry of molecular complexes of several trigonal donor molecules with the trigonal acceptor 1,3,5trinitrobenzene, TNB, is reported. Generally, replacement of a moiety by another of similar shape and size does not change the overall packing. The 1:1:1 (triphenylisocyanurate).(TNB).(benzene) solvate is isostructural to the corresponding $1: 1: 1$ thiophene solvate, confirming the so-called benzene-thiophene exchange rule. The 1:1 complex of tris-2,4,6-(4-methylphenyl)-1,3,5-triazine and TNB is layered and the layers have quasi-trigonal symmetry. The triazine ring may be replaced by a phenyl ring without any change in the crystal structure. Thus, 1,3,5-tris(4-methylphenyl)benzene and TNB form an isostructural 1:1 complex. Such shape/ size exchange may be further explored in the 1:1 complex of 1,3,5-tris[5-(2-chlorothienyl)]benzene and TNB. Here both phenylthienyl and chloro-methyl exchanges are simultaneously possible and yet another isostructural complex is obtained. Finally, the $1: 1$ complex of 1,3,5-tris(2-thienyl)benzene and TNB is also found to have a very similar structure. However, when 1,3,5-triphenylbenzene and TNB are taken in 1:1 ratio in solution, the result is a 1:3 molecular complex. This is unexpected in view of the phenyl-thienyl exchange rule, and some rationalisation is provided for the unusual formation of this 1:3 complex. Many of these structures are pertinent from the viewpoint of carry-over of trigonal molecular symmetry into the crystal, a contemporary theme in the engineering of crystal structures for octupolar non-linear optical applications, while ready access to the 1,3,5-trisubstituted benzenes for this study was made possible by a general and efficient protocol for the synthesis of these compounds from the corresponding acetyl aromatics.
\end{abstract}

\section{Introduction}

We have been investigating the relationship between molecular and crystal symmetry in the context of the engineering of crystal structures for octupolar non-linear optical applications. Ever since the work of Kitaigorodskii, it has been well-known that save for the centre of inversion, molecular symmetry need not be carried over into the crystal. ${ }^{1}$ In the octupolar context, trigonal symmetry is particularly relevant ${ }^{2,3}$ and whilst several trigonal molecules adopt trigonal and hexagonal crystal symmetries (or pseudosymmetric variants of these), there are many others which do not. Some combination of substituent size and awkwardness of molecular shape seems to be required for a $C_{3}$-sym-

Keywords: crystal engineering; shape/size effects; close-packing; benzenethiophene exchange; chloro-methyl exchange; trinitrobenzene; trimerisation; acetylthiophene; $\mathrm{SiCl}_{4}$. metry molecule to adopt trigonal or quasi-trigonal crystal symmetry. ${ }^{4}$ Identifying or selecting such combinations is still a matter of instinct and personal bias. So, any reliable trends in the packing preferences of these compounds are expected to be useful in crystal engineering studies. In this paper, we describe the crystal structures of molecular complexes of several trigonal donor molecules with the trigonal acceptor 1,3,5-trinitrobenzene, TNB, and where replacement of a substituent(s) by others with a similar shape and size does not change the overall packing. These efforts have been greatly aided by the development of a general and simple protocol for the synthesis of symmetrical 1,3,5-trisubstituted benzenes, especially thienyl substituted compounds. ${ }^{5}$ In crystal synthesis, effective mapping of crystal packing depends crucially on an ability to manipulate molecular structure at will. This so-called molecular-supramolecular balance in crystal engineering needs to be carefully planned so that synthetic targets are both interesting and attainable. $^{6,7}$ 
<smiles>O=c1n(-c2ccccc2)c(=O)n(-c2ccccc2)c(=O)n1-c1ccccc1</smiles><smiles>Cc1ccc(-c2nc(-c3ccc(C)cc3)nc(-c3ccc(C)cc3)n2)cc1</smiles>

TMT<smiles>Cc1ccc(-c2cc(-c3ccc(C)cc3)cc(-c3ccc(C)cc3)c2)cc1</smiles>

TMB<smiles>Clc1ccc(-c2cc(-c3ccc(Cl)s3)cc(-c3ccc(Cl)s3)c2)s1</smiles>

TCIThB<smiles>c1csc(-c2cc(-c3cccs3)cc(-c3cccs3)c2)c1</smiles>

TThB<smiles>c1ccc(-c2cc(-c3ccccc3)cc(-c3ccccc3)c2)cc1</smiles>

\section{Results and Discussion}

\section{Benzene-thiophene exchange}

Our earlier work dealt with $C_{3}$-symmetrical isocyanurates and triazines and the crystal chemistry of the pure compounds was reported. ${ }^{8,9}$ In this study, we decided to co-crystallise some of these substances with TNB. The first system of choice was therefore TNB:1,3,5-triphenylisocyanurate (TPI). Crystallisation of these compounds in a 1:1 ratio from benzene yielded the 1:1:1 (TPI) (TNB). (benzene) solvate. The structure of this solvate is shown in Fig. 1. Table 1 gives the main crystallographic information. The TPI molecules are assembled with respectably good $\mathrm{C}-\mathrm{H} \cdots \pi$ hydrogen bonds $\left(2.63 \AA\right.$, $158^{\circ}$, H-atoms neutron normalised, interaction $\mathbf{a}$ in the figure) as centrosymmetric dimers. ${ }^{10}$ Most interestingly, there is a quintupledecker sandwich of phenyl groups, TNB molecules and a central molecule of benzene which also lies on a centre of symmetry. This sandwich is held together with the $\pi \cdots \pi$ interactions b $(3.76 \AA)$ and $\mathbf{c}(3.40 \AA)$, these distances being the average of the perpendicular distances between the centroid of one of the molecules to the plane of the other. This procedure for estimating the stacking distance was followed because the TPI, TNB and benzene rings are all non-parallel.

Whilst the structure of the (TPI).(TNB).(benzene) complex is of low symmetry, it provided a lead in terms of examining the so-called benzene-thiophene exchange phenomenon. It was noted that the structure is built around the central benzene molecule which is essential to this particular mode of assembly. The question then arose as to whether this structure would be preserved if TPI and TNB were cocrystallised from thiophene. The structural relationship
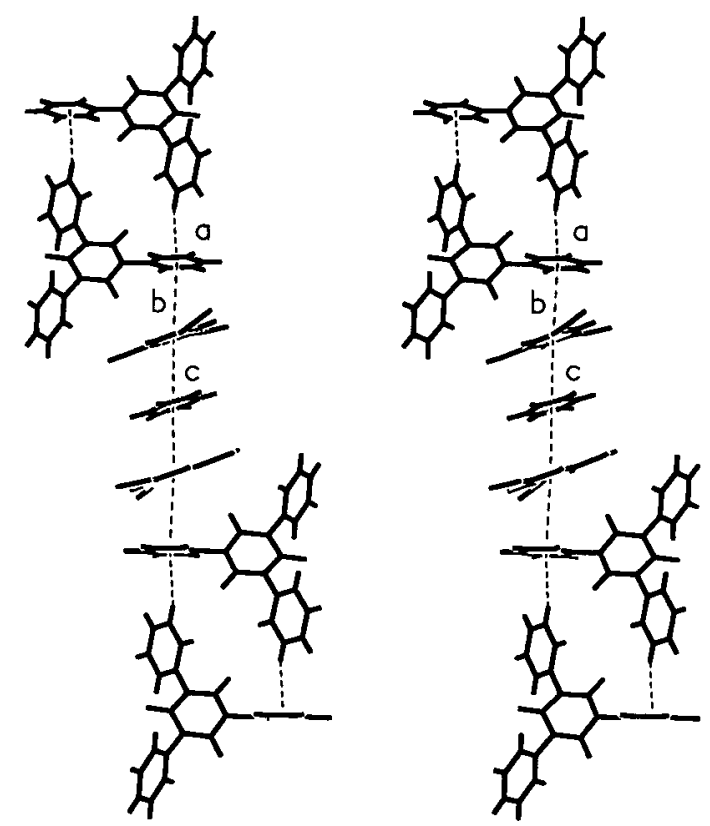

Figure 1. Stereoview of the quintuple-sandwich structure of the 1:1:1 complex (TPI).(TNB)-(benzene). For a description of the non-bonded contacts $\mathbf{a}, \mathbf{b}$, and $\mathbf{c}$, see the text. Notice that the numbers of the various molecules in this figure do not reflect the overall stoichiometry of the molecular complex. 
Table 1. Crystallographic data for the compounds in this study

\begin{tabular}{|c|c|c|c|c|c|c|c|}
\hline & $(\mathrm{TPI}) \cdot(\mathrm{TNB}) \cdot($ benzene $)$ & $(\mathrm{TPI}) \cdot(\mathrm{TNB}) \cdot($ thiophene $)$ & $(\mathrm{TMT}) \cdot(\mathrm{TNB})$ & $(\mathrm{TMB}) \cdot(\mathrm{TNB})$ & $(\mathrm{TClThB}) \cdot(\mathrm{TNB})$ & $(\mathrm{TThB}) \cdot(\mathrm{TNB})$ & $(\mathrm{TPB}) \cdot(\mathrm{TNB})$ \\
\hline Emp. form & $\mathrm{C}_{30} \mathrm{H}_{21} \mathrm{~N}_{6} \mathrm{O}_{9}$ & $\mathrm{C}_{29} \mathrm{H}_{20} \mathrm{~N}_{6} \mathrm{O}_{9} \mathrm{~S}_{0.5}$ & $\mathrm{C}_{30} \mathrm{H}_{24} \mathrm{~N}_{6} \mathrm{O}_{6}$ & $\mathrm{C}_{33} \mathrm{H}_{27} \mathrm{~N}_{3} \mathrm{O}_{6}$ & $\mathrm{C}_{24} \mathrm{H}_{12} \mathrm{Cl}_{3} \mathrm{~N}_{3} \mathrm{O}_{6} \mathrm{~S}_{3}$ & $\mathrm{C}_{24} \mathrm{H}_{15} \mathrm{~N}_{3} \mathrm{O}_{6} \mathrm{~S}_{3}$ & $\mathrm{C}_{42} \mathrm{H}_{27} \mathrm{~N}_{9} \mathrm{O}_{18}$ \\
\hline Form. wt & 609.53 & 612.54 & 564.55 & 561.58 & 640.99 & 537.57 & 945.73 \\
\hline$T(\mathrm{~K})^{\mathrm{a}}$ & $293(2)$ & $293(2)$ & $120(2)$ & $120(2)$ & $120(2)$ & $293(2)$ & $120(2)$ \\
\hline Cryst. syst. & triclinic & triclinic & triclinic & triclinic & triclinic & triclinic & triclinic \\
\hline Space group & $P \overline{1}$ & $P \overline{1}$ & $P \overline{1}$ & $P \overline{1}$ & $P \overline{1}$ & $P \overline{1}$ & $P \overline{1}$ \\
\hline$a(\AA)$ & $11.448(2)$ & $11.426(2)$ & $8.762(2)$ & $7.1630(14)$ & $7.2150(14)$ & $14.234(3)$ & $7.2634(15)$ \\
\hline$b(\AA)$ & $11.866(2)$ & $11.806(2)$ & $11.966(2)$ & 14.801(3) & $13.847(3)$ & $14.416(3)$ & $15.714(3)$ \\
\hline$c(\AA)$ & $12.441(2)$ & $12.423(3)$ & $12.956(3)$ & 14.972(3) & $14.723(3)$ & 14.494(3) & $18.290(4)$ \\
\hline$\alpha(\mathrm{deg})$ & $71.82(3)$ & $72.32(3)$ & $104.64(3)$ & $115.18(3)$ & $115.50(3)$ & $119.60(3)$ & $76.96(3)$ \\
\hline$\beta(\mathrm{deg})$ & $63.26(3)$ & $63.14(3)$ & $93.22(3)$ & $98.28(3)$ & 103.12(3) & $96.86(3)$ & $86.28(3)$ \\
\hline$\gamma(\mathrm{deg})$ & $88.31(3)$ & $88.42(3)$ & $93.75(3)$ & $102.4(3)$ & $94.94(3)$ & $102.92(3)$ & $86.11(3)$ \\
\hline$Z$ & 2 & 2 & 2 & 2 & 2 & 4 & 2 \\
\hline$V(\AA)^{3}$ & $1421.8(5)$ & $1412.6(5)$ & $1307.7(5)$ & $1352.3(5)$ & $1264.4(4)$ & $2426.2(8)$ & $2026.4(7)$ \\
\hline$D_{\text {calc }}\left(\mathrm{Mg} / \mathrm{m}^{3}\right)$ & 1.421 & 1.440 & 1.434 & 1.379 & 1.683 & 1.472 & 1.550 \\
\hline$F(000)$ & 630 & 632 & 588 & 588 & 648 & 1104 & 972 \\
\hline$\theta$ range & $1.82-27.50$ & $1.83-27.53$ & $1.76-27.88$ & $2.71-27.48$ & $2.80-27.88$ & $1.52-24.98$ & $2.66-27.51$ \\
\hline \multirow[t]{3}{*}{ Index range } & $0 \leq h \leq 14$ & $0 \leq h \leq 14$ & $0 \leq h \leq 11$ & $0 \leq h \leq 9$ & $0 \leq h \leq 9$ & $0 \leq h \leq 16$ & $0 \leq h \leq 9$ \\
\hline & $-15 \leq k \leq 15$ & $-15 \leq k \leq 15$ & $-15 \leq k \leq 15$ & $-19 \leq k \leq 18$ & $-18 \leq k \leq 17$ & $-17 \leq k \leq 16$ & $-20 \leq \mathrm{k} \leq 20$ \\
\hline & $-13 \leq l \leq 16$ & $-13 \leq l \leq 16$ & $-17 \leq l \leq 16$ & $-19 \leq l \leq 18$ & $-19 \leq l \leq 18$ & $-17 \leq l \leq 17$ & $-23 \leq 1 \leq 23$ \\
\hline$R 1$ & 0.0411 & 0.0518 & 0.0535 & 0.0597 & 0.0473 & 0.0705 & 0.0513 \\
\hline$w R 2$ & 0.1179 & 0.1340 & 0.1659 & 0.1687 & 0.1332 & 0.1082 & 0.1240 \\
\hline GOF & 1.078 & 1.073 & 1.075 & 1.107 & 1.026 & 1.067 & 1.057 \\
\hline $\mathrm{N}$-total ${ }^{\mathrm{b}}$ & 6515 & 6484 & 6193 & 6187 & 5942 & 8518 & 9236 \\
\hline $\mathrm{N}$-indep $\mathrm{p}^{\mathrm{c}}$ & 6515 & 6484 & 6193 & 6187 & 5942 & 8518 & 9236 \\
\hline $\mathrm{N}-$ obsd $^{\mathrm{d}}$ & 4685 & 4124 & 5050 & 4852 & 4750 & 2796 & 7294 \\
\hline Variables & 407 & 444 & 380 & 379 & 352 & 660 & 622 \\
\hline
\end{tabular}

a Temperature of the data collection.

${ }^{b} \mathrm{~N}$-total is the total number of reflections collected.

${ }^{\mathrm{c}} \mathrm{N}$-indep is the number of independent reflections.

${ }^{\mathrm{d}} \mathrm{N}$-obsd. is the number observed reflections based on the criterion $\mathrm{I}>2 \sigma_{\mathrm{I}}$

between benzene and thiophene has been long noted. The similarities of the phenyl and thienyl groups in both shape and size were utilised by Green and Schmidt to prepare solid solutions of 1-phenyl-4-(2',6'-dichlorophenyl)-1,3-butadiene and 1-thienyl-4-( $2^{\prime}, 6^{\prime}$-dichlorophenyl)-1,3-butadiene. ${ }^{11}$ The complete solid state solubility of 4,5-phenanthrylene disulphide in pyrene attests to the importance of close-packing nature of the S-atom in crystals. $^{12}$ More recent studies have attempted to
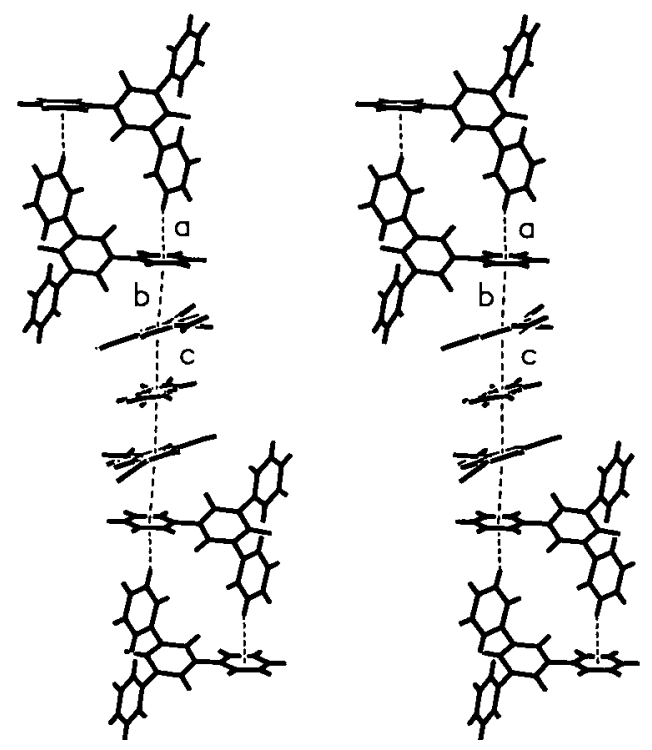

Figure 2. Stereoview of the complex 1:1:1 complex (TPI).(TNB).(thiophene). The thiophene molecule sits on an inversion centre and is disordered. Note the close similarity to Fig. 1. examine this phenomenon further ${ }^{13}$ but no completely satisfactory crystallographic study of an isostructural benzene-thiophene (or phenyl-thienyl) pair of compounds has been reported.

In the present instance, we found that the (TPI).(TNB). (thiophene) is isostructural to the benzene solvate. Figs. 1 and 2 are nearly identical. The corresponding interactions are a $\left(\mathrm{C}-\mathrm{H} \cdots \pi: 2.62 \AA, 157.6^{\circ}\right), \mathbf{b}(\pi \cdots \pi: 3.77 \AA)$ and $\mathbf{c}$ $(\pi \cdots \pi: 3.43 \AA)$. To complete this near identity, the thiophene molecule is disordered about a centre of symmetry so that it mimics the benzene molecule (Fig. 3). We attempted to co-crystallise TPI and TNB from benzenethiophene mixtures. Crystals containing both solvents were obtained but we could not obtain reliable estimates

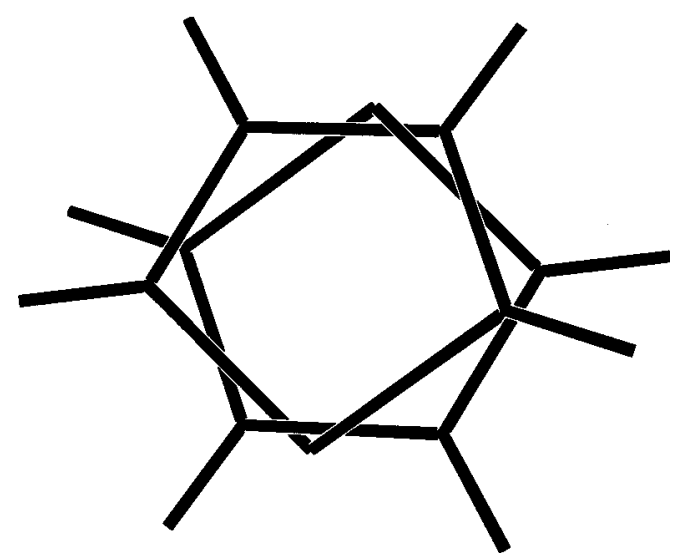

Figure 3. Mimicry between a disordered thiophene molecule and a benzene. See Figs. 1 and 2. 


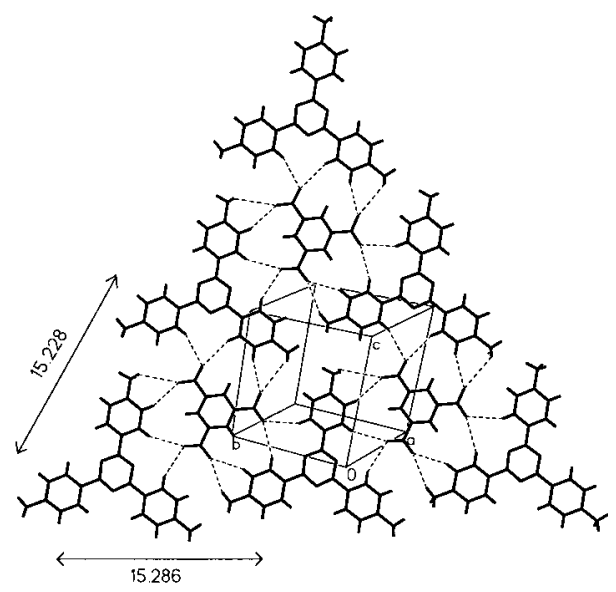

Figure 4. Quasi-trigonal layer structure of the 1:1 complex (TMT).(TNB). The $\mathrm{C}-\mathrm{H} \cdots \mathrm{O}$. hydrogen bonds are indicated.

of the benzene:thiophene ratio in these crystals with NMR. To summarise, it appears then that a thioether S-atom is a reasonable supramolecular surrogate for a $\mathrm{CH}=\mathrm{CH}$ moiety and that the pair of solvates described above validates the benzene-thiophene exchange rule.

\section{Quasi-trigonal layered structures}

The major aim of the study being the generation of trigonal crystal structures from $C_{3}$-symmetry molecules, we next crystallised a 1:1 mixture of TNB and tris-2,4,6-(4-methylphenyl)-1,3,5-triazine, TMT from chlorobenzene, the TMT itself being obtained in a straightforward way from 4-methyl-1-cyanobenzene. The result was a 1:1 molecular complex (TMT) $\cdot(\mathrm{TNB})$ with quasi-trigonal symmetry in two dimensions (Fig. 4). The structure is layered and the sheet in Fig. 4 corresponds to $(22-2)$ with the pseudotrigonal translational vectors 15.228 and $15.286 \AA$ marked in. It is pertinent to state here that neither TMT or TNB yield trigonal (or quasi-trigonal) layers in their native crystal structures. There is a dense system of $\mathrm{C}-\mathrm{H} \cdots \mathrm{O}$ hydrogen bonds (range $2.49-2.91 \AA, 156-141^{\circ}$ ), not unusual in

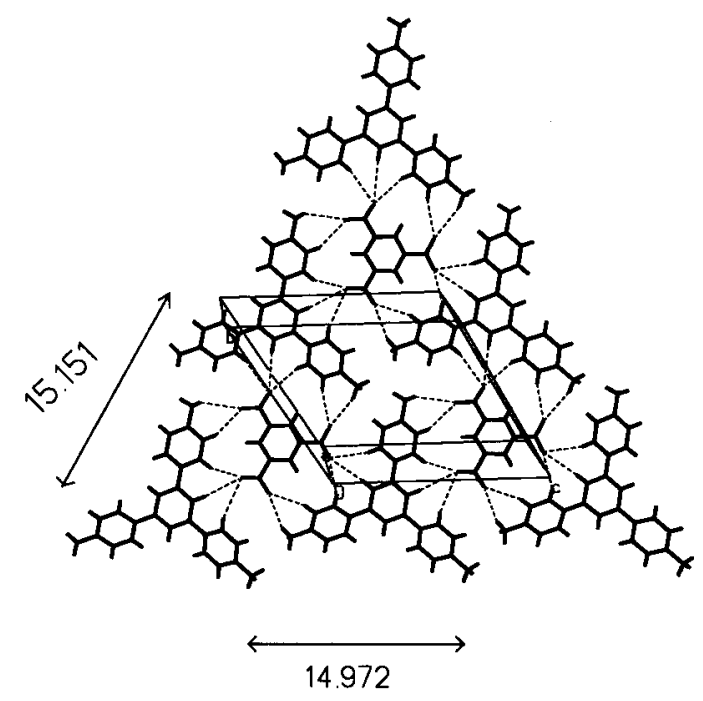

Figure 5. Layer structure of the 1:1 complex (TMB).(TNB). Compare this with Fig. 4 and note the equivalence of the triazine and phenyl rings. molecular complexes of TNB. Adjacent layers are centrosymmetrically related and stacked with a $\pi \cdots \pi$ separation of $3.71 \AA$, so that immediate NLO applications are not apparent. Because of the nature of the stacking, however, the structure is not trigonal (or quasi-trigonal) in three dimensions.

The next step was to attempt a substitution of the triazine ring in TMT with the nearly isosteric phenyl ring, namely to crystallise TNB with 1,3,5-tris(4-methylphenyl)benzene, TMB. Even the contemplation of such a strategy was only possible because TMB is readily accessed in high yields from 4-methylacetophenone via a literature procedure. ${ }^{14}$ The crystal structure of the 1:1 complex (TMB).(TNB) is shown in Fig. 5 from which it may be seen that it resembles the (TMT) $\cdot(\mathrm{TNB})$ complex (Fig. 4) very closely. The same system of $\mathrm{C}-\mathrm{H} \cdots \mathrm{O}$ hydrogen bonds (range $2.49-2.96 \AA$, $148-154^{\circ}$ ) is found with the layers corresponding to the (2 -2 0) planes. The translational vectors within these planes are 15.151 and $14.972 \AA$ and are very similar to those in (TMT) $\cdot(\mathrm{TNB})$. Further, adjacent layers are stacked in an anti-parallel fashion across centres of symmetry $(\pi \cdots \pi$ $3.35 \AA$ A) as before.

Noting that this system was amenable to a triazine-benzene exchange without a change in crystal packing, we next attempted a slightly more ambitious perturbation. 1,3,5tris[5-(2-chlorothienyl)]benzene, TClThB, was prepared from 2-acetyl-5-chlorothiophene by trimerisation with $\mathrm{SiCl}_{4}$ and co-crystallised with TNB to give the 1:1 complex. The structure of (TClThB).(TNB) is shown in Fig. 6 from which it may be seen that it is isostructural to (TMB).(TNB) and therefore to (TMT).(TNB). In this structure, the layers lie along $(2-20)$ and are assembled with a few $\mathrm{C}-\mathrm{H} \cdots \mathrm{O}$ hydrogen bonds and a weak $\mathrm{C}-\mathrm{H} \cdots \mathrm{Cl}$ interaction $(2.96 \AA$, $\left.140^{\circ}\right)$. The similarity between the translational vectors (14.819 and 14.723 $\AA$ ) along the pseudo-trigonal directions and the corresponding vectors in (TMT).(TNB) and (TMB).(TNB) show the near identity in shape and size of the TMT, TMB and TCIThB molecules. The modification of TMB to TClThB involves two shape/size substitutions-a switch of a phenyl ring to a thienyl ring and a switch of a

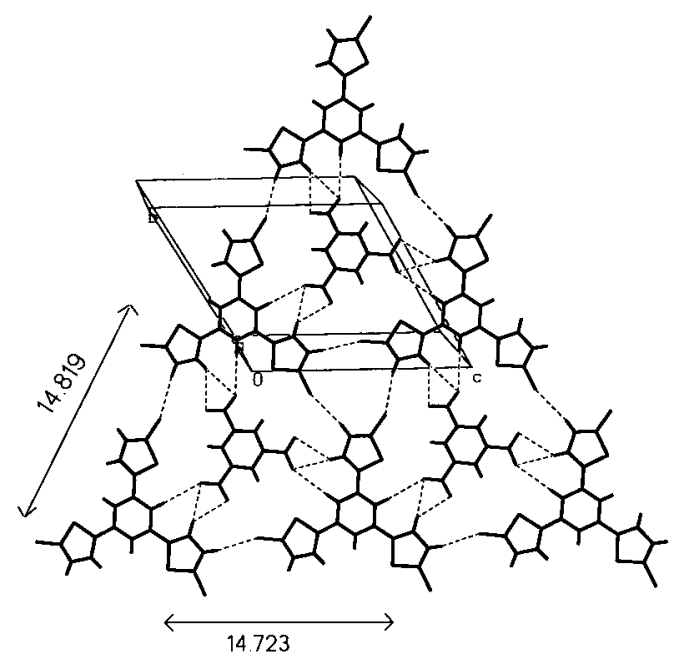

Figure 6. Layer structure of the 1:1 complex (TClThB) $(\mathrm{TNB})$ which is equivalent to the layer in (TMB) (TNB) shown in Fig. 5. 


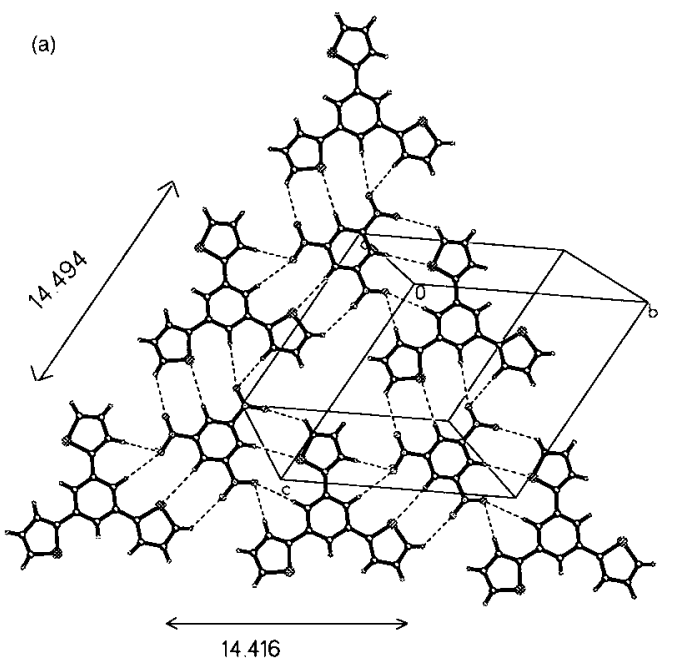

(b)

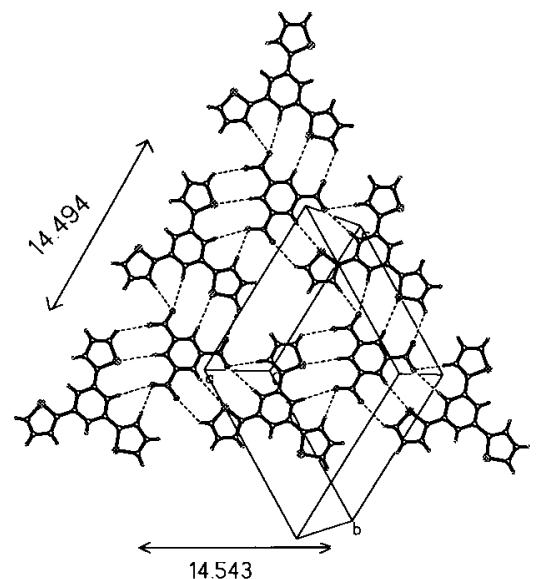

Figure 7. (a) Layer structure in the $1: 1$ complex (TThB).(TNB). Notice the 3-point synthon I constituted with $\mathrm{C}-\mathrm{H} \cdots \mathrm{O}$ and $\mathrm{C}-\mathrm{H} \cdots \mathrm{S}$ hydrogen bonds. (b) Corresponding symmetry-independent layer which is related to that in Fig. 7a by a pseudo-inversion centre.

methyl group to a chloro group. ${ }^{15}$ Both the benzenethiophene and the chloro-methyl exchange rules are with precedent but in this pretty example, it has been possible to make both switches simultaneously without any change in overall packing and crystal structure. This uncommon occurrence $^{16}$ is a sure sign that shape and size factors, namely close-packing non-directional arguments hold sway in this family of crystal structures. Accordingly, it may be noted that there are no short and/or particularly directional S-atom contacts within the layer. The layers themselves are centrosymmetrically stacked as before $(\pi \cdots \pi 3.30 \AA)$.

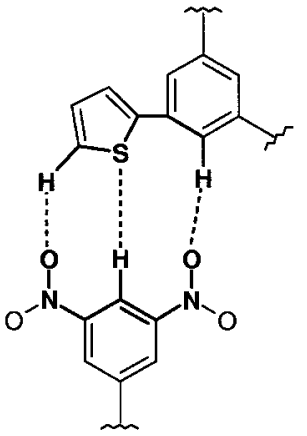

I

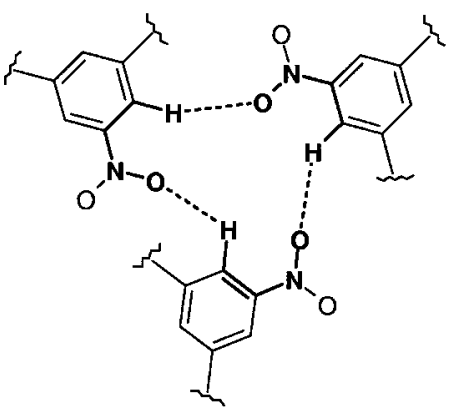

II
Finally, we co-crystallised the analogously synthesised 1,3,5-tris(2-thienyl)benzene, TThB, and TNB to obtain yet another quasi-trigonal layered structure (Fig. 7a). This layer is smaller in its dimensions. The quasi-trigonal translations are 14.494 and $14.416 \AA$ which is around $3 \%$ less than the corresponding vectors in the three complexes above. This shrinkage follows from the smaller dimensions of the TThB molecule when compared to TMT, TMB and TClThB and is unexceptional. What is important, however, is that the layer structure is topologically related to the previous cases. A novel feature is the appearance of the three-point synthon $\mathbf{I}$ constituted with $\mathrm{C}-\mathrm{H} \cdots \mathrm{O}$ and $\mathrm{C}-\mathrm{H} \cdots \mathrm{S}\left(2.99 \AA, 165.9^{\circ}\right)$ hydrogen bonds. There is a hint of a specific role for the $\mathrm{S}$-atom here with the $\mathrm{C}-\mathrm{H}$ group aligned along the extension of one of the $\mathrm{C}-\mathrm{S}$ covalent bonds of the thienyl ring-exactly the direction predicted by the nucleophileelectrophile model of Parthasarathy. ${ }^{17}$ Fig. $7 \mathrm{~b}$ shows a symmetry-independent and almost identical layer (translations 14.494 and $14.543 \AA$; C-H..S, $2.93 \AA$ A, $160.1^{\circ}$ ) related by pseudo-inversion to the layer in Fig. 7a. Stacking of the $\pi \cdots \pi$ type between layers related by true (3.41, $3.47 \AA$ ) and pseudo inversion centres (3.39 $\mathrm{A}$ ) completes the structure.

\section{1:3 Molecular complex of 1,3,5-triphenylbenzene (TPB) and TNB}

In the light of these observations on quasi-trigonal layered structures, we were intrigued to note that when TPB and TNB were crystallised from $1: 1 \mathrm{CCl}_{4}$ EtOAc in 1:1 proportions, the result was a 1:3 molecular complex of the two constituents, followed by the appearance of crystals of pure (unused) TPB. There was no evidence of any 1:1 complex. Fig. 8 shows the layer structure and Fig. 9 is a stereoview of the stacking $(\pi \cdots \pi, 3.42,3.37,3.37 \AA)$ between the layers in (TPB) $(\mathrm{TNB})_{3}$. The molecules of TNB are arranged according to the trimer synthon II, and the general shape and size of the TNB trimer is nearly equal to that of the TPB molecule. Clearly, this fortuitous equivalence favours the formation of the 1:3 complex, but why then is this structure

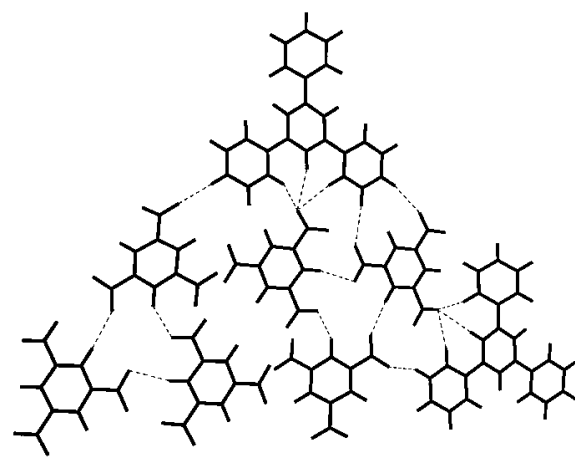

Figure 8. Layer structure in the $1: 3$ complex (TPB).(TNB) $)_{3}$. Notice the trimer synthon II formed by the TNB molecules and its equivalence in shape and size to the TPB molecule. 

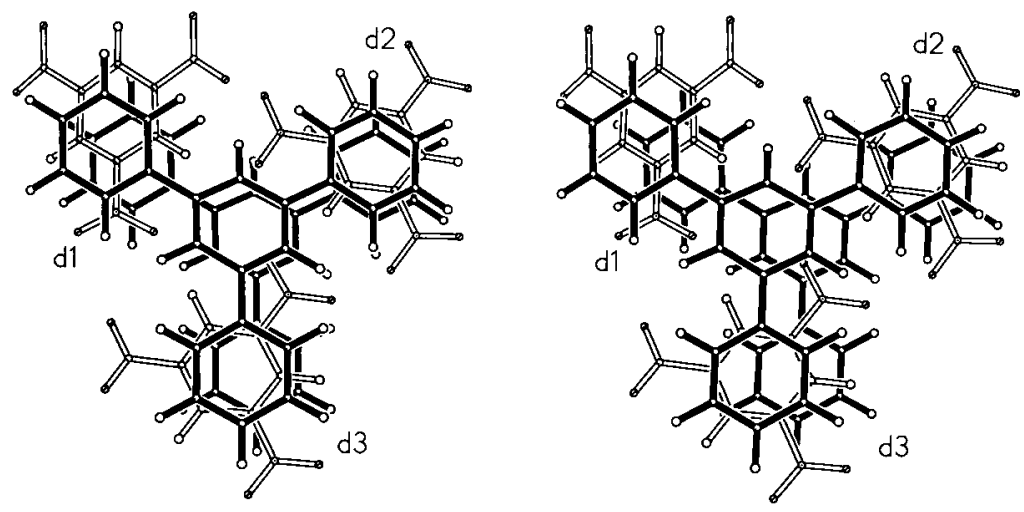

Figure 9. Stereoview of the stacking arrangement of the TNB-trimer and the TPB molecule in the 1:3 complex (TPB).(TNB) $)_{3}$. The stacking distance are d1, 3.41 ; d2, 3.37; d3, $3.37 \AA$

not formed by TThB? After all, the dimensions of the TThB and TPB molecules must be nearly the same according to the phenyl-thienyl exchange rule. It would appear that the formation of the 1:1 complex for TThB and TNB, and of the 1:3 complex for TPB and TNB is the result of subtle yet specific factors. We can only speculate here on a few of these factors: (a) Certain $\mathrm{S}$-atom specific contacts in (TThB) $(\mathrm{TNB})$ say the $\mathrm{C}-\mathrm{H} \cdots \mathrm{S}$ interaction are not possible for (TPB).(TNB); (b) stacking interactions could be more effective between phenyl and TNB rings than between thienyl and TNB rings, given the different ring*..ring spacings in the two cases $(3.40 \AA$ versus $3.60 \AA)$; and (c) slight size and shape differences between the benzene and thiophene moieties are unimportant in the pair of molecules TClThB (volume $311 \AA^{3}$ ) and TMB $\left(341 \AA^{3}\right.$ ) which show benzene-thiophene exchange, but cross a critical limit in the smaller pair of molecules TThB $\left(264 \AA^{3}\right)$ and TPB $\left(290 \AA^{3}\right)$ which do not.

Geometrical factors are, however, important because we noted that: (a) packing calculations $\left(\text { Cerius }^{2}\right)^{18}$ on $(\mathrm{TThB}) \cdot(\mathrm{TNB})$ and a simulated structure in which TPB is placed in the TThB sites showed unfavourable conformations and geometries for the TPB molecules. There is not much variation in density, $C_{k}$ and cell volume for the experimental and simulated structures. However, the lower packing energy per cell in the latter case could be due to the poorer overall packing which is reflected in the considerably higher value of the occupiable but inaccessible volume in the unit cell $\left(8.37 \AA^{3}\right.$ in the simulated structure versus $0.52 \AA^{3}$ in the experimental structure); (b) $d-\theta$ plots for (TPB) $\cdot(\mathrm{TNB})_{3}$ and pure TNB showed that $\mathrm{C}-\mathrm{H} \cdots \mathrm{O}$ interactions are far more significant in the latter, hinting that overall threefold pseudosymmetry and close-packing are important in the former. We are now attempting to obtain a 1:3 complex of TThB and TNB to probe this (lack of) phenyl-thienyl exchange further.

\section{Conclusions}

Trigonal molecules do not always yield crystal structures with trigonal or quasi-trigonal symmetry. However, within a small family of molecular complexes of 1,3,5-trinitrobenzene with symmetrical 1,3,5-trisubstituted aromatics, layered structures are generally formed with two- dimensional quasi-trigonal symmetry. The replacement of either the hub moiety or the radial substituents in the trigonal donor components of these complexes, by groups of the same shape and size, leaves the crystal structures largely unaltered. This study provides a good confirmation of the so-called benzene-thiophene exchange rule for crystal structures. Such shape and size rules operate in crystal structures governed by close-packing factors. It is also seen that an ability to access new organic molecules through general and simple protocols greatly enhances the scope of the crystal engineering exercise.

\section{Experimental}

Melting points were recorded on Labhosp or Veego melting point apparatus and are uncorrected. Infrared spectra were recorded on Nicolet Impact-400 FT IR spectrometer. All samples were recorded as $\mathrm{KBr}$ wafers unless mentioned otherwise. Ultraviolet spectra were recorded on Shimadzu UV 2100 or UV $260.300 \mathrm{MHz}{ }^{1} \mathrm{H}$ NMR spectra were recorded on Brucker spectrometer. Samples were made in chloroform-d solvent and chemical shifts were reported in $\delta$ scale using tetramethylsilane as the internal standard. Coupling constants $J$ are in hertz $(\mathrm{Hz})$. Analytical thinlayer chromatography (TLC) were performed on $\left(10 \times 5 \mathrm{~cm}^{2}\right)$ glass plates coated with TLC grade silica gel (acme's; 100-200 mesh). Flash chromatography was performed using silica gel. For all the reactions dry magnesium sulphate was used as drying agent after work-up. Yields refer to chromatographically isolated yields. Thiophene, 2-acetyl-5-chlorothiophene were purchased from Lancaster Synthesis. $\mathrm{SiCl}_{4}$ was obtained from Aldrich.

General procedure for trimerisation of acetyl derivatives. ${ }^{14}$ To a solution of the acetyl derivative $(40 \mathrm{mmol})$ in absolute $\mathrm{EtOH}(40 \mathrm{ml})$ was added $\mathrm{SiCl}_{4}$ (2-4 equiv.) dropwise with stirring at $0^{\circ} \mathrm{C}$ and the reaction mixture was stirred at ambient temperature. At the conclusion of reaction (TLC), the dark reaction mixture was poured into ice-cold water and extracted with dichloromethane $(3 \times 30 \mathrm{ml})$. The combined organic extract was washed with water, brine and then dried. Evaporation of the solvent and purification of the crude product by column chromatography (silica gel) using hexane as a eluent furnished the trimerized product. 
1,3,5-Tris(4-methylphenyl)]benzene (TMB). ${ }^{14}$ 4-Methylacetophenone $(1.5 \mathrm{~g}, 11.19 \mathrm{mmol})$ in absolute $\mathrm{EtOH}(10 \mathrm{ml})$ was treated with $\mathrm{SiCl}_{4}(2.5 \mathrm{~g}, 14.8 \mathrm{mmol})$ according to the general procedure described above for $6 \mathrm{~h}$ to produce TMB as a white solid $(1 \mathrm{~g}, 84 \%)$. mp: $176-178^{\circ} \mathrm{C}$ (Lit mp: $177-$ $\left.178^{\circ} \mathrm{C}\right) .{ }^{1} \mathrm{H}$ NMR $\left(300 \mathrm{MHz}, \mathrm{CDCl}_{3}\right): \delta 2.41(\mathrm{~s}, 9 \mathrm{H}), 7.28$ $(1 / 2 \mathrm{ABq}, J=7.9 \mathrm{~Hz}, 6 \mathrm{H}), 7.58(1 / 2 \mathrm{ABq}, J=8.0 \mathrm{~Hz}, 6 \mathrm{H})$, $7.73(\mathrm{~s}, 3 \mathrm{H})$

1,3,5-Tris[5-(2-chlorothienyl)]benzene (TCIThB). ${ }^{5} \quad$ 2Acetyl-5-chlorothiophene (300 mg, $1.87 \mathrm{mmol})$ in absolute EtOH $(6 \mathrm{ml})$ was treated with $\mathrm{SiCl}_{4}(1.3 \mathrm{~g}, 7.85 \mathrm{mmol})$ according to the general procedure described above for $12 \mathrm{~h}$ to give TClThB as a white solid $(159 \mathrm{mg}, 60 \%)$. $\mathrm{mp}: 177-179^{\circ} \mathrm{C}$. IR (KBr): $\nu_{\max } 746,786,854,992$, 1454, 1538, $2927 \mathrm{~cm}^{-1}$. UV $\left(\mathrm{CHCl}_{3}\right): \lambda_{\max }=304 \mathrm{~nm}$, $\epsilon=52595 \mathrm{M}^{-1} \mathrm{~cm}^{-1} .{ }^{1} \mathrm{H}$ NMR $\left(300 \mathrm{MHz}, \mathrm{CDCl}_{3}\right): \delta 6.93$ $(\mathrm{d}, J=4.0 \mathrm{~Hz}, 3 \mathrm{H}), 7.15(\mathrm{~d}, J=3.7 \mathrm{~Hz}, 3 \mathrm{H}), 7.50(\mathrm{~s}, 3 \mathrm{H}) .{ }^{13} \mathrm{C}$ NMR $\left(75.43 \mathrm{MHz}, \mathrm{CDCl}_{3}\right): \delta 122.0,123.3,127.3,130.2$, 135.3, 141.5. Anal.: for $\mathrm{C}_{18} \mathrm{H}_{9} \mathrm{~S}_{3} \mathrm{Cl}_{3}$ Calcd: 50.53 (C), 2.12 (H); Found: $49.99(\mathrm{C}), 2.07(\mathrm{H})$.

1,3,5-Tris(2-thienyl)benzene $\quad\left(\right.$ TThB) ${ }^{5,19} \quad$ 2-Acetylthiophene $(5 \mathrm{~g}, 40 \mathrm{mmol})$ in absolute $\mathrm{EtOH}(40 \mathrm{ml})$ was treated with $\mathrm{SiCl}_{4}(13.6 \mathrm{~g}, 80 \mathrm{mmol})$ according to the general procedure described above for $6 \mathrm{~h}$ to deliver TThB as a white solid $(1.8 \mathrm{~g}, 42 \%)$. mp: $154-155^{\circ} \mathrm{C}$ (Lit. mp: $156-$ $\left.158^{\circ} \mathrm{C}\right) . \mathrm{IR}(\mathrm{KBr}): \nu_{\max } 699,820,840,860,1038,1236$, $1590, \quad 2924 \mathrm{~cm}^{-1}$. UV $\left(\mathrm{CHCl}_{3}\right): \quad \lambda_{\max }=296 \mathrm{~nm}$, $\epsilon=50359 \mathrm{M}^{-1} \mathrm{~cm}^{-1} .{ }^{1} \mathrm{H}$ NMR $\left(300 \mathrm{MHz}, \mathrm{CDCl}_{3}\right): \delta 7.11$ $\left(\mathrm{dd}, J_{1}=J_{2}=3.7 \mathrm{~Hz}, 3 \mathrm{H}\right), 7.33\left(\mathrm{dd}, J_{1}=1.3, J_{2}=1.1 \mathrm{~Hz}, 3 \mathrm{H}\right)$, $7.41\left(\mathrm{dd}, J_{1}=1.1 \mathrm{~Hz}, J_{2}=1.2 \mathrm{~Hz}, 3 \mathrm{H}\right), 7.73(\mathrm{~s}, 3 \mathrm{H}) .{ }^{13} \mathrm{C}$ NMR $\left(75.43 \mathrm{MHz}, \mathrm{CDCl}_{3}\right): \delta 122.8,123.9,125.4,128.2$, 135.7, 143.6. Mass: m/e $324\left(\mathrm{M}^{+}\right)$. TThB has been synthesised in the literature ${ }^{19}$ via a palladium mediated crosscoupling reaction but our method is much simpler.

1,3,5-Tris(phenyl)benzene (TPB). ${ }^{14}$ Acetophenone $(5.24 \mathrm{~g}$, $43.6 \mathrm{mmol})$ in absolute $\mathrm{EtOH}(30 \mathrm{ml})$ was treated with $\mathrm{SiCl}_{4}$ $(10.4 \mathrm{~g}, 61.1 \mathrm{mmol})$ according to the general procedure described above for $6 \mathrm{~h}$ to furnish 4 as a white solid (3.8 g, 86\%). mp: $171-172^{\circ} \mathrm{C}$ (Lit. mp: $173^{\circ} \mathrm{C}$ ). ${ }^{1} \mathrm{H}$ NMR $\left(300 \mathrm{MHz}, \mathrm{CDCl}_{3}\right): \delta 7.35-7.41(\mathrm{~m}, 3 \mathrm{H}), 7.44-7.50(\mathrm{~m}$, $6 \mathrm{H}), 7.67-7.71(\mathrm{~m}, 6 \mathrm{H}), 7.78(\mathrm{~s}, 3 \mathrm{H})$.

(TPI) $\cdot($ TNB) $\cdot($ benzene) 1:1:1 complex. Pale yellow needle- and diamond-shaped crystals of the complex (mp $283-285^{\circ} \mathrm{C}$ ) were obtained by crystallisation of equimolar amounts of TPI $(18 \mathrm{mg})$ and TNB $(11 \mathrm{mg})$ from $10 \mathrm{ml}$ of benzene. IR (KBr): $\nu_{\max } 694,1344,1406,1491,1543,1622$, $1701,3072 \mathrm{~cm}^{-1}$.

(TPI)·(TNB)・(thiophene) 1:1:1 complex. Pale yellow needle-shaped crystals of the complex (mp $283-285^{\circ} \mathrm{C}$ ) were obtained by crystallisation of equimolar amounts of TPI $(18 \mathrm{mg})$ and TNB $(11 \mathrm{mg})$ from $10 \mathrm{ml}$ of thiophene. IR $(\mathrm{KBr}): \nu_{\max } 694,1344,1415,1491,1543,1622,1699$, $3072 \mathrm{~cm}^{-1}$.

(TMT) $\cdot($ TNB) 1:1 complex. Pale yellow needles $\left(\mathrm{mp}>300^{\circ} \mathrm{C}\right)$ were obtained by crystallisation of equimolar amounts of TMT $(18 \mathrm{mg})$ and TNB $(11 \mathrm{mg})$ from $10 \mathrm{ml}$ of chlorobenzene IR (KBr): $\nu_{\max } 850,910,1068,1338,1365$, $1408,1545,1622,3109 \mathrm{~cm}^{-1}$.

(TMB) (TNB) 1:1 complex. Pale yellow needles (mp 208$220^{\circ} \mathrm{C}$ ) were obtained by crystallisation of equimolar amounts of TMB (17 mg) and TNB (11 mg) from $10 \mathrm{ml}$ of chlorobenzene. IR (KBr): $\nu_{\max } 812,914,1066,1338$, $1543,1618,3109 \mathrm{~cm}^{-1}$.

(TCIThB) $\cdot($ TNB $)$ 1:1 complex. Pale orange needles (mp $160-162^{\circ} \mathrm{C}$ ) were obtained by crystallisation of equimolar amounts of TClThB $(21 \mathrm{mg})$ and TNB $(11 \mathrm{mg})$ from $10 \mathrm{ml}$ of $\mathrm{CCl}_{4}$. IR (KBr): $\nu_{\max } 785,852,991,1059,1190,1454$, $1529,1591 \mathrm{~cm}^{-1}$.

(TThB) •(TNB) 1:1 complex. Pale orange needles (mp 125$127^{\circ} \mathrm{C}$ ) were obtained by crystallisation of equimolar amounts of TThB $(16 \mathrm{mg})$ and TNB $(11 \mathrm{mg})$ from $10 \mathrm{ml}$ of $\mathrm{CCl}_{4}$. IR (KBr): $\nu_{\max } 700,1342,1545,1596,1620 \mathrm{~cm}^{-1}$.

(TPB) -(TNB) 1:3 complex. Pale yellow needles of the 1:3 complex (mp $146-148^{\circ} \mathrm{C}$ ) were obtained initially when of

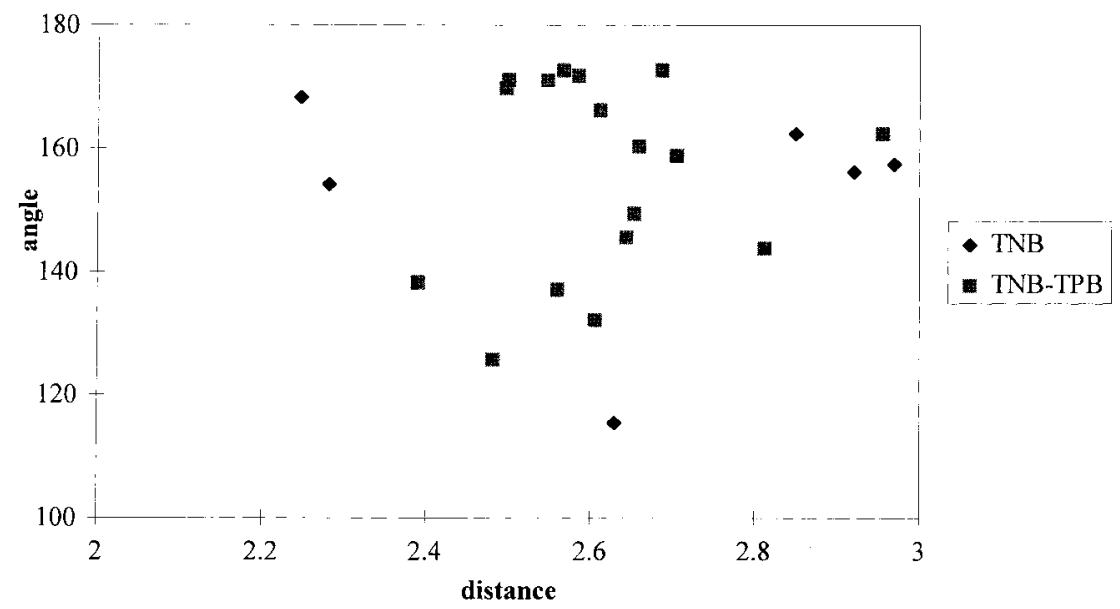

Figure 10. $d-\theta$ scatterplot for $\mathrm{C}-\mathrm{H} \cdots \mathrm{O}$ hydrogen bonds in (TPB) $(\mathrm{TNB})_{3}$ (grey squares) and pure TNB (black diamonds). Notice the short, linear contacts in the latter structure. These $\mathrm{C}-\mathrm{H} \cdots \mathrm{O}$ hydrogen bonds are more important in pure TNB than in the molecular complex. 
equimolar amounts of TPB (15 mg) and TNB (11 mg) were taken in $10 \mathrm{ml}$ of $1: 1 \mathrm{CCl}_{4}$.EtOAc. Subsequently, colourless needles of the excess TPB crystallised out of the solution. IR. $(\mathrm{KBr}): \nu_{\max } 729,765,914,1074,1344,1547,1622$, $3133 \mathrm{~cm}^{-1}$.

X-Ray diffraction data were collected on an Enraf-Nonius FAST area detector with a rotating anode source at 120(2)K in Philadelphia, or on an Enraf-Nonius CAD4 diffractometer at room temperature in Hyderabad. None of the data sets were corrected for absorption effects, these being deemed to be of minor consequence. The solution of the structures for all the complexes were carried out with the SHELXS $97^{20}$ program and the refinements were carried out with the SHELXL $97^{20}$ program on Silicon Graphics workstations. Pertinent details of the data collection, structure solution and refinement are given in Table 1 for the seven crystal structures in this study. The CIF files for these structures have been deposited with the Cambridge Crystallographic Data Centre. The $\mathrm{C}-\mathrm{H}$...O bonds in these structures are conveniently analysed with scatterplots of hydrogen bond distance, $d$ versus hydrogen bond angle, $\theta$. Such a $d-\theta$ plot is shown in Fig.10. The packing energy calculations were performed with the Smart Minimiser module $\left(\right.$ Cerius $\left.^{2}\right)$ using the Burchart 1.01-Dreiding 2.21 forcefield with the charges obtained with the chargeequilibration method.

\section{Acknowledgements}

We thank the CSIR for the fellowship support (P. K. T.) and RSIC-Mumbai for recording the spectral data (K. C.; S. K.). H. L. C. acknowledges financial suppport from grant CA-10925 of the National Institutes of Health. G. R. D. acknowledges financial support from CSIR under project 01/1570/99/EMR-II.

\section{References}

1. Kitaigorodskii, A. I. Molecular Crystals and Molecules, Academic: New York, 1973.
2. Zyss, J.; Nicoud, J. -F. Curr. Opin. Solid State Mater. Sci. 1996, $1,533-546$.

3. Wolff, J. J.; Gredel, F.; Oeser, T.; Irngartinger, H.; Pritzkow, H. Chem. Eur. J. 1999, 5, 29-38.

4. Desiraju, G. R. In Comprehensive Supramolecular Chemistry, MacNicol, D. D., Bishop, R., Toda, F., Eds.; Pergamon: New York, 1996; Vol. 6, pp 1-22.

5. Kotha, S.; Chakraborty, K.; Brahmachary, E. Synlett. 1999, $1621-1623$.

6. Nangia, A.; Desiraju, G. R. Top. Curr. Chem. 1998, 198, 57-95. 7. Nangia, A.; Desiraju, G. R. Current Challenges on Large Supramolecular Assemblies; In NATO ARW Series, Tsoucaris, G., Ed.; Kluwer: Dordrecht, 1999, pp 193-208.

8. Thalladi, V. R.; Brasselet, S.; Bläser, D.; Boese, R.; Zyss, J.; Nangia, A.; Desiraju, G. R. Chem. Commun. 1997, 1841-1842.

9. Thalladi, V. R.; Brasselet, S.; Weiss, H. -C.; Bläser, D.; Katz, A. K.; Carrell, H. L.; Boese, R.; Zyss, J.; Nangia, A.; Desiraju, G. R. J. Am. Chem. Soc. 1998, 120, 2563-2577.

10. Desiraju, G. R.; Steiner, T. The Weak Hydrogen Bond in Structural Chemistry and Biology, Oxford University Press: New York, 1999 (pp 152-158).

11. Elgavi, A. J.; Green, B. S.; Schmidt, G. M. J. J. Am. Chem. Soc. 1973, 95, 2058-2059.

12. Sloan, G. J.; McGhie, A. R. Techniques of Chemistry, Wiley: New York, 1988 (p 29).

13. Etter, M. C.; Parker, D. L.; Ruberu, S. R.; Panunto, T. W.; Britton, D. J. Inclus. Phenom. Mol. Recognit. Chem. 1990, 8, 395407.

14. Elmorsy, S. S.; Pleter, A.; Smith, K. Tetrahedron Lett. 1991, $32,4175-4176$.

15. Desiraju, G. R.; Sarma, J. A. R. P. Proc. Ind. Acad. Sci. (Chem. Sci.). 1986, 96, 599-605.

16. Kálmán, A.; Párkányi, L.; Argay, G. Acta Crystallogr. 1993, B49, 1039-1049.

17. Rosenfield, R. E.; Parthasarathy, R.; Dunitz, J. D. J. Am. Chem. Soc. 1977, 99, 4860.

18. Cerius $^{2}$ Program, Molecular Simulations, 9685 Scranton Road, San Diego, CA 92121-3752 (USA) and 240/250 The Quorum, Barnwell Road, Cambridge CB5 8RE (UK).

19. Pleter, A.; Jenkins, I.; Jones, D. E. Tetrahedron 1997, 53, 10357-10400.

20. Sheldrick, G. M. SHELX-97: Package for Crystal Structure Solution and Refinement, University of Göttingen, Germany. 1997. 